\title{
Letters
}

\section{Preventing coronary heart disease}

High baseline risk strategy and cost effectiveness of guidelines

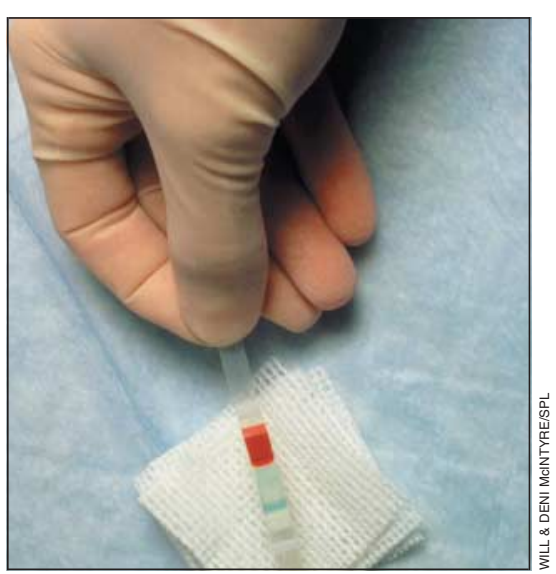

EDITOR-Manuel et al found, contrary to Rose's population health strategy, that a high baseline risk strategy was the most effective approach to coronary heart disease prevention with a statin in Canada. ${ }^{1}$ This approach is consistent with new UK guidelines. ${ }^{2}$ Using data from the nationally representative Scottish health survey 1998 we estimate that $25 \%$ of $40-74$ year olds in Scotland would be eligible for primary prevention of cardiovascular disease with a statin when using this approach, with large implications for prescribing budgets. The annual cost in Scotland of treating all those eligible with $40 \mathrm{mg}$ generic simvastatin would be $£ 40 \mathrm{~m}$ ( $€ 58 \mathrm{~m}$; $\$ 69.8 \mathrm{~m}$ ) and would be substantially higher with branded statins.

In the Scottish health survey 2003, 51\% of men and $49 \%$ of women aged 55-64 years had high blood pressure and the figure rose to $77 \%$ for men and women aged 75 years and older. Treating these patients with a thiazide diuretic would be an effective way of reducing mortality ${ }^{3}$ and would be more cost effective than statins. ${ }^{4}$ To date there has been no trial evidence of an additional reduction in all cause mortality with statins in patients with thiazide treated hypertension, even in the ASCOT trial. ${ }^{5}$

We believe that cost effectiveness of guidelines should be considered before their introduction with comparison to other, potentially more cost effective, approaches to the primary prevention of cardiovascular disease. In addition to lifestyle measures including weight management and smoking cessation, these might include treatment with low dose bendrofluazide if blood pressure is found to be raised. In middle aged and elderly patients this would bring widespread population benefits, reminiscent of Rose's approach, and is likely to be considerably more affordable and cost effective than using statins.

D Graham Mackenzie specialist registrar Public Health Department, NHS Fife, Leven, Fife KY8 5RR gm@nhs.net

John Forbes reader in health economics Sarah Wild senior lecturer in public health School of Clinical Sciences and Community Health, University of Edinburgh, Edinburgh EH8 9AG

Philip Rutledge consultant in medicines management Public Health and Health Policy, Lothian NHS Board, Edinburgh EH8 9RS

Competing interests: None declared.

1 Manuel DG, Lim J, Tanuseputro P, Anderson GM, Alte DA, Laupacis A et al Revisiting Rose: strategies for reducing coronary heart disease. BMJ 2006;332:659-62 (18 ing cor

2 Wood D, Wray R, Poulter N, Williams, Kirby M, Patel V, et al. JBS 2: Joint British Societies' guidelines on prevention of cardiovascular disease in clinical practice. Heart

3 Wright JM, Lee CH, Chambers GK. Systematic review of antihypertensive therapies: Does the evidence assist in antihypertensive therapies: Does the evidence
choosing a first-line drug? CMAJ 1999;161:25-32.

Scottish Intercolleginte Guidelines Network SIGN gide line. Lipids and the primarde prave Networ. SIGN guideine. Lipids and the primary prevention of coronary hear Tollege Sevisians, 199

B, Poulter NR, Wedel H, Beevers G, Caulfield M, et al. Prevention of coronary and stroke events with atorvastatin in hypertensive patients who have verage or lower-than-average cholesterol concentrations the Anglo-Scandinavian cardiac outcomes trial-lipi owering arm (ASCOT-LLA): a multicentre randomised

\section{Patients are people, not cases}

EDITOR-Everyone knows the importance of seeing patients as people, not cases. But the idea seems to have made a less successful transfer in terms of risk reduction.

Lowering some factor by a small percentage in everyone-regardless of level of risk-requires seeing everyone as a disease waiting to happen. ${ }^{12}$ It requires people with no reason for concern to make unnecessary adjustments to their lives in yet another area. And it requires doctors to provide more warnings about potential problems, even as the time available for the truly sick becomes more limited.

Joan McClusky medical writer

New York, NY 10003, USA

joanmcclusky@interlaceglobal.com

Competing interests: None declared.
1 Jackson R, Lynch J, Harper S. Preventing coronary heart disease. BMJ 2006;332:617-8. (18 March.)

2 Manuel DG, Lim J, Tanuseputro P, Anderson GM, Alter DA, Laupacis A, et al. Revisiting Rose: strategies for reducing coronary heart disease. BMJ 2006;332:659-62. (18 March.)

Blood pressure lowering in elderly patients with stroke

EDITOR-Mant et al say that the results of PROGRESS are not applicable to the stroke population in the community. ${ }^{1}$ One reason cited is that patients in the community were 12 years older than those recruited to PROGRESS. The authors explain the dangers of aggressive blood pressure lowering for people older than 80 and recommend further urgent studies.

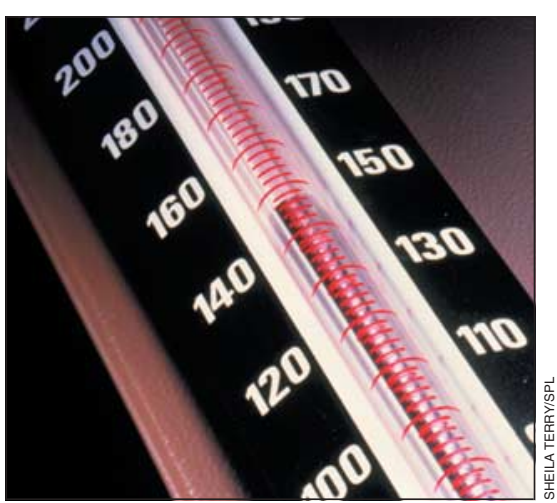

Guidance on blood pressure lowering in people older than 80 is still lacking, ${ }^{2}$ but this is no reason to deprive this group of appropriate treatment. If, for example, a fit and active 81 year old woman has a minor stroke then it seems appropriate to treat hypertension aggressively. It may be unethical for this patient to be recruited to a placebo arm of a blood pressure lowering trial and be deprived of treatment that may prevent a stroke.

It is thus simply not possible to perform randomised controlled trials in all groups of patients. Moreover, most studies actually ask more questions than they answer. For many cases, treatment is decided following discussion between patient and doctor, with the aid of the information provided by randomised controlled trials.

Elliot Epstein consultant physician elliotepstein832@hotmail.com

Anil Kumar specialist registrar, general and geriatric medicine

Walsall Manor Hospital NHS Trust, Walsall, West Midlands WS2 9PS

Competing interests: None declared. 
1 Mant J, McManus R, Hare R. Applicability to primary care of national clinical guidelines on blood pressure lowering of national clinical guidelines on blood pressure lowering
for people with stroke: cross sectional study. BMJ or people with stroke:

2 Williams B, Poulter NR, Brown MJ, Davis M, et al. The BHS guidelines working party guidelines for management of hypertension: report of the fourth working party of the British Hypertension Society, 2004-BHS IV.J Hum Hypertens 2004;18:139-85.

\section{Estimated glomerular filtration rate needs UK consensus}

EDITOR-Chronic kidney disease imposes a substantial health burden, affecting 5\% of the population. The renal national service framework recommends using the estimated glomerular filtration rate as a more sensitive marker for chronic kidney disease than serum creatinine. The quality and outcomes framework 2006-7 requires the estimated rate to achieve six points for a chronic kidney disease register and 21 points for measuring and treating blood pressure in chronic kidney disease

In December 2005, 55 out of 58 UK clinical biochemistry departments contacted agreed to be interviewed about current and proposed reporting of estimated glomerular filtration rate and methods for estimated glomerular filtration rate and creatinine. Seventeen $(31 \%)$ were providing some degree of reporting (median 9 (range 1-24) months). Three routinely reported the rate. The remaining centres provided a limited service to named consultants and general practitioners.

The estimated glomerular filtration rate was calculated by abbreviated (four variable) modified diet in renal disease (MDRD) equation (15 centres), ${ }^{2}$ the MDRD equation (one centre), ${ }^{3}$ and the Cockcroft and Gault equation (one centre). ${ }^{4}$ Serum creatinine was measured by the kinetic Jaffe method in all these laboratories by using different analytical platforms. One centre routinely corrected the estimated glomerular filtration rate for ethnicity, nine provided no correction, two provided the correction if ethnicity was stated, and five provided a related comment.

Of $38(69 \%)$ centres not providing reporting, five had no plans to provide the service, 24 were planning to start within 12 months. These centres cited information technology problems, concerns over the workload of renal physicians, and confusion concerning the calculation of the rate as reasons for not introducing reporting.

Routine reporting of estimated glomerular filtration rate will become a necessity, but the current provision will be unable to support the quality and outcomes framework for chronic kidney disease. Recommendations are urgently required to standardise the approach to calculate estimated glomerular filtration rate. We believe that the Renal Association's guidance of using the abbreviated formula for modified diet in renal disease should be adopted..$^{5}$ In the longer term, the measure- ment of serum creatinine should be standardised.

Annice Mukherjee specialist registrar in diabetes and endocrinology

annicemukherjee@hotmail.com

Donal O'Donaghue co-chair renal advisory group for renal NSF implementation

Aram Redenski consultant chemical pathologist

John New consultant physician and diabetologist

Salford Royal NHS Trust, Hope Hospital, Salford M6 8HD

Patrick Twomey consultant chemical pathologist Ipswich Hospital, Ipswich IP4 5PD

Competing interests: None declared.

1 NHS Employers. The new QOF areas and indicators. http://www.nhsemployers.org/primary/primary-

Levey AS, Greene T, Kusek JW, Beck GJ, Group MS. A simplified equation to predict glomerular filtration rate from serum creatinine. J Am Soc Nephrol 2000;11:A0828.

3 Levey AS, Bosch JP, Lewis JB, Greene T, Rogers N, Roth D. A more accurate method to estimate glomerular filtration A more accurate method to estimate glomerular filtration rate from serum creatinine: a new prediction equation. Intern Med 1999;130:461-70.

4 Cockcroft DW, Gault MH. Prediction of creatinine clearance from serum creatinine. Nephron 1976;16:31-41. clearance from serum creatinine. Nephron 1976;16:31-41. 5 Chronic kidney disease in adults: UK guidelines for identification, management and referral of adults 23 Mar 2006).

\section{Grieving the death of a child}

Clinicians should ensure that lethal drugs are handed back

EDITOR-Davies's case reports of completed suicide in recently bereaved parents are a timely reminder of one of the few areas in suicide prevention (access to lethality) where clinicians can intervene to save lives. ${ }^{1}$

Within the anger of acute grief-case 1 describes "holding on" to the decreased person beyond the usual grief experience ${ }^{1}-$ the child's drugs can acquire a symbolic value. The same applies to bereaved spouses who may also come to see their late partner's tablets as hope that betrayed them. Gunnell and Lewis provide a useful conceptual framework in which to consider a person's risk of suicide $^{2}$ : a combination of predisposing plus facilitating factors minus protective factors such as social role, parenthood, help seeking behaviours during crisis, and religious belief. In the context of the sudden loss of many protective factors, where most people's coping skills would be overwhelmed by the loss of a child, clinicians must identify access to lethal overdose.

To ask grieving parents to "hand over the medications" in the immediate aftermath of a death may seem unthinkable, but preparation for this should be part of the anticipatory grieving process. ${ }^{3}$ One solution would be for carers to sign for controlled drugs when home care is being arranged: "These drugs are for the treatment of $\mathrm{X}$ and must be returned to (named key worker) if $\mathrm{X}$ leaves this address." For other lethal drugsfor example, insulin and cardiac drugswritten consent from carers formalises their safe return in the event of death or other change in circumstances. As Davies points out, relatives with high suicidal intent are likely to deny this, making psychiatric assess- ment unreliable during the immediate aftermath of a death. There are lessons here for all hospital and community practitioners.

Peter Byrne liaison psychiatrist

Oldchurch Hospital, Romford RM7 0BE

p.byrne@ucl.ac.uk

Competing interests: None declared.

1 Davies DE. Parental suicide after the expected death of a child at home BMJ 2006;332:647-8. (18 March.)

2 Gunnell D, Lewis S. Studying suicide from the life course perspective: implications for prevention. Br J Psychiatry 2005; $187: 206-8$

Raphael B. Grieving the death of a child. $B M$ 2006;332:620-1. (18 March.)

Supporting health professionals who care for grieving patients may benefit all

EDITOR-Davies outlined two cases of palliative care in the community for children where mothers committed suicide after the death of their children by using the remaining opioid drugs. ${ }^{1}$ Supporting healthcare professionals who work in palliative care, and particularly in such circumstances, could be as helpful for them as it may be for their patients. Published work has recognised that patients' suicide has an impact on psychiatrists. $^{23}$ The qualitative study in which I am currently participating (which includes senior house officers (SHOs) in psychiatry) has identified the traumatic effect of suicide risk assessment on the SHOs when the patient subsequently attempted or committed suicide. The emotional effects of such experiences have a lasting influence on the SHOs' practice by denting their professional confidence. The feedback from the focus group interviews that I have conducted so far includes comments on the helpful aspects of sharing such experiences and the feelings of guilt and being blamed.

In the accompanying editorial, Raphael addressed the complex issue of supporting parents whose child is dying from a terminal illness and the complexities of grief in such circumstances. ${ }^{4}$ Despite recognising the invaluable role of such support she seems to be unconvinced by the evidence for providing bereavement support for families and calls for "controlled trials" that would inform the practice. The medical profession should not need controlled trials to justify such care. Anyone who is working with grieving people will benefit from education and training and from more informal ongoing support in their work place. All this will inform professionals about the complex psychological reaction to loss, facilitate empathy, improve the therapeutic relationship, and ultimately be helpful to the patients. Sharing such experiences in peer reviewed journals, as Davies has done, is for the benefit of all who work in health care.

Svetlin V Vrabtchev specialist registrar in

psychotherapy

Cedar House, Blackberry Hill Hospital, Bristol BS16 2EG

svetlin.vrabtchev@awp.nhs.uk

Competing interests: None declared.

1 Davies DE. Parental suicide after the expected death of a child at home. BMJ 2006;332:647-8. (18 March.) 
2 Alexander DA, Klein S, Gray NM, Dewar IG, Eagles JM. Suicide by patients: questionnaire study of its effect on Suicide by patients: questionnaire study of its
consultant psychiatrists. BMJ 2005;320:1571-4.

3 Ruskin R, Sakinofsky MD, Bagy RM, Dickens S, Sousa ( Impact of patient suicide on psychiatrists and psychiatric
trainees. Acad Psychiatry 2004:28:104-10.

of a child. BMJ 2006.332:620-1. (18 March)

\section{Gluteal injections in increasingly obese population}

\section{Needle length for intramuscular} injections

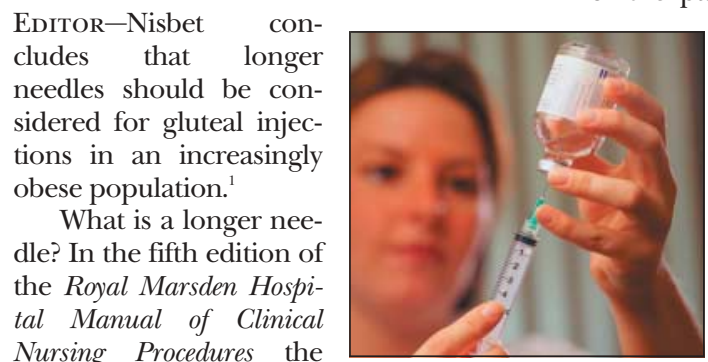

However, the gluteal region is not the most practical intramuscular injection site in those aged 25-65. These patients do not lie still in bed. They either sit, stand, walk around, or prefer to be injected in other sites.

Thirdly, the gluteal region is the more often used site for intramuscular injections in children, who are held by their parents, and this age group was excluded from the study.

Fourthly, non-ambulant and elderly patients often have atrophy of the gluteal muscles, and they represent the age group of the patients who receive intramuscular injections in the gluteal region most often. This age group was excluded from the study. Surprisingly, age was not correlated with dorsogluteal depth, for reasons not mentioned in this study.

Although the study tries to address an important issue, it does not show the true picture.

Saurabh Rai vascular research fellow authors suggest the following needle lengths, based on patient weight, for gluteal intramuscular injections ${ }^{2}$ :

31.5-40.00 kg: $2.5 \mathrm{~cm}$ needle

40.5-90.00 kg: 5-7.5 cm needle

$>90 \mathrm{~kg}: 10-15 \mathrm{~cm}$ needle.

They cite Lenz."

\section{Brian J Anthony lecturer}

Middlesex University, Archway Campus, London

N19 5LW

b.anthony@mdx.ac.uk

Competing interests: None declared.

1 Nisbet AC. Intramuscular gluteal injections in the increasingly obese population: retrospective study. BMJ ingly obese population:

2 Mallett J, Dougherty L. Royal Marsden Hospital manual of clinical nursing procedures. 5th ed. Oxford: Blackwell clinical nursing

3 Lenz CL. Make your needle selection right to the point. Nursing 1983;13:50-1.

\section{Essential considerations for intramuscular injections}

EDITOR-We agree with Nisbet that standard available needles may be inappropriate for gluteal intramuscular injections, especially when the incidence of obesity is increasing. ${ }^{1}$ However, his paper seems to exaggerate the problem.

Firstly, there are other preferred sites for intramuscular injections-for example, the mid-deltoid, rectus femoris, and vastus lateralis. ${ }^{2}$ Simpler clinical variables such as the weight of the patient, muscle mass of the injection site, and the amount of subcutaneous fat should be assessed for choosing the correct needle length. ${ }^{3}$

In this study the sole criterion for the assessment of subcutaneous fat at gluteal region was the need to undergo the computed tomography scans of the pelvis without mentioning the indication for which this was performed. Without knowing the underlying indication it is hard to estimate how many of these patients would have required gluteal intramuscular injections.

Secondly, antiemetics and analgesics are the common drugs given intramuscularly.
University Hospital Birmingham NHS Trust, Selly Oak Hospital, Birmingham B29 6JD saurabh_rai@hotmail.com

Nidhi Nandan senior house officer Royal Shrewsbury Hospital, Shrewsbury SY3 8XQ

Competing interests: None declared.

1 Nisbet AC. Intramuscular gluteal injections in the increasngly obese population: retrospective study. $B M$ 2006;332:637-8. (18 March.) 1999;13:47-52

Lenz CL. Make your needle selection right to the point Nursing 1983;13:50-1.

\section{Time to make paracetamol with methionine available}

EDITOR-What happened to the provision of "safe" paracetamol that had been campaigned for-that containing a judicious amount of methionine, which reduced the potential for overdose?

It was released for public purchase some years ago, being designed to save lives, such as those mentioned in Tanne's article, ${ }^{1}$ but it seems to have disappeared from the few pharmacies' shelves that stocked it seemingly reluctantly. I say reluctantly because I and several others made an effort to check its availability for public purchase and were astounded that most pharmacists when asked for it either kept it "under the counter" or "had to chase it up and would take a couple of days to obtain it"-and in almost every case the purchaser was asked why he or she would wish to purchase it when there was some perfectly safe paracetamol without methionine for sale already.

If "safe" paracetomol is available, and so many people are dying of paracetamol poisoning who could be saved through the widespread sale of "safe" paracetamol," why does the government's health department fail to ensure its widespread availability?

John P Heptonstall director

Morley Acupuncture Clinic, Leeds LS27 8EG

john@mac-tcm.demon.co.uk
2 Workman B. Safe injection techniques. Nurs Stand
Competing interests: None declared.

1 Tanne J. Paracetamol causes most liver failure in UK and US. BMJ 2006;332:628-a. (18 March.)

\section{Googling your treatment for analgesia in labour}

EDITOR-Giustini described how Google has become the most powerful influence in searching the internet. ${ }^{1}$ In a recent observational study on the labour ward at University College Hospital, London, we found that $33 \%$ of pregnant women used the internet as a source of information about labour pain relief. This is a change from the results of a previous study conducted five years ago in Liverpool, in which the internet had been used by less than 3\% of pregnant women. ${ }^{2}$

We wondered what expectant mothers would find on "Googling your treatment." When searching for health information, patients are known to look only at the first few links after their search, and very few of them later remember from which websites they retrieved information or by whom the sites were created. ${ }^{3}$

We feared that the one third of pregnant women using the internet were accessing information that was inaccurate and potentially misleading their labour analgesia choices. We performed a search at the main Google UK site (not Google Scholar), using "labour pain relief" as our criterion. The search provided "about 3300000 results," of which we examined the top 10 .

Six were written by or had direct contribution from a doctor, two were articles in peer reviewed journals, one was by a midwife, and one was a commercial website selling TENS machines. All of the noncommercial websites were informative and of high quality, providing balanced information. We considered the information to be anaesthetist friendly, and we were reassured by our investigation.

In our internet savvy society, we suggest that healthcare providers google their particular topic to discover what kind of information their patients are finding. A cautionary note: as search engines continuously update the pages to which they link and their ranking, we advise regularly repeating the search to keep up with the dynamic flux of the internet.

James Holding research fellow james.holding@uclh.nhs.uk

Michael V Holmes foundation year 1 trainee in anaesthesia

Centre for Anaesthesia, University College

Hospital, London NW1 2BU

Competing interests: None declared.

\footnotetext{
Giustini D. How Google is changing medicine. $B M$ 2005:331:1487-8. (24 December)

2 Barclay PM. Book review: www.painfreebirthing.com. $\mathrm{BrJ}$ Anaesthesia 2003;90: 816 .

3 Eysenbach G. Köhler C. How do consumers search for and appraise health information on the world wide web? Qualitative study using focus groups, usability tests, and in-depth interviews. BMJ 2002;324:573-7. (9 March.)
} 


\section{Neonatal lupus erythematosus is not always benign}

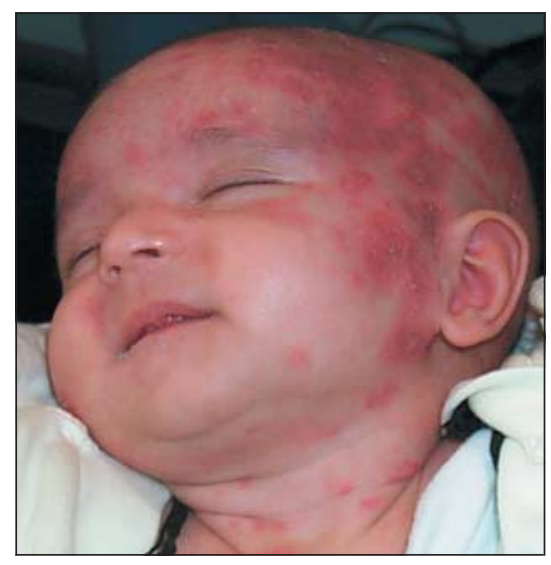

EDIToR-The clinical case described by Dennes and Nelson-Piercy in Minerva (with photograph) described a baby with neonatal cutaneous lupus. ${ }^{1}$

This is a benign and self limiting condition when it is present in isolation, but it should be emphasised that patients with neonatal lupus erythematosus have a substantial risk of congenital heart block, a potentially fatal complication. Neonatal lupus erythematosus accounts for $80 \%$ of all cases of congenital heart block. ${ }^{2}$ About half of the reported cases of neonatal lupus erythematosus have skin disease and half have congenital heart block, with $10 \%$ having both. ${ }^{2}$ Congenital heart block is permanent and requires a pacemaker in many cases. It also results from the passive transfer of maternal autoantibodies, anti-RoSSA and anti-La/SSB as in the cutaneous manifestations. Therefore, all babies with neonatal cutaneous lupus should be investigated for congenital heart block. ${ }^{3}$

Andrew G Affleck specialist registrar in dermatology Queen's Medical Centre, Nottingham NG7 2UH andyaffleck@doctors.org.uk

Competing interests: None declared.

1 Minerva. BMJ 2006;332:676. (18 March)

2 Boh EE. Neonatal lupus erythematosus. Clin Dermatol 2004;22:125-8.

Buyen JP, Rupel A, Clancy RM. Neonatal lupus syndromes. Lupus 2004;13:705-12.

\section{Independent sector treatment centres: experience and spin}

EDITOR-Independent sector treatment centres were recently praised by the Department of Health. ${ }^{1}$ Ophthalmologists have highlighted the problems experienced and predicted for cataract centres. ${ }^{2-4}$ The government argument that independent centres provide value for money in elective surgery is unconvincing because it considers "spot purchasing" costs of "waiting list initiatives" rather than the costs of planned NHS care. Procurement may drain the NHS of funds that might otherwise be invested in comprehensive care in NHS hospitals.

Clinical quality in some ophthalmology schemes has also been a concern. ${ }^{23}$ Furthermore, cataract waiting times had come down before the mobile cataract independent sector treatment centres became operational. ${ }^{5}$

"Putting patients in charge of where they are treated means that all providers have to compete and this competition helps drive a patient-centred service."1 But offering uncoordinated choice at fixed tariffs may make service planning and the financial viability of NHS providers difficult. Unlimited choice must be expensive. Experience suggests most patients want quick access to good local facilities. Cooperation-for example in clinical networks-rather than competition is thought to drive improved clinical quality. Quality may become a hostage to market forces when the bottom line is profit.

To date, no training in ophthalmology has been provided in independent sector treatment centres. No innovation has emerged from those for cataracts-rather, overseas teams have had to improve their standards to reach NHS ophthalmology standards. ${ }^{23}$ The much trumpeted innovation of mobility is of uncertain benefit to patients who often have to travel further to reach these mobile units than to receive conventional care locally. High volume NHS cataract surgery operating lists can, and often do, achieve the same numbers of patients treated per session as in the mobile units. However, NHS units also provide holistic and comprehensive ophthalmology care to all comers, and clinical training.

Reform and "self improvement" of NHS services is also urged. ${ }^{1}$ However, most of the process redesign now claimed existed in initiatives such as the "Action on Cataract" schemes. ${ }^{25}$ These self improvements are testimony to both the innovative skills of NHS ophthalmologists and the close collaboration between hospital and primary care. These professional leadership qualities may be lost in the fragmentation of the current procurement of the independent sector centres.

Simon P Kelly consultant ophthalmologist Bolton Hospitals NHS Trust, Bolton BL4 OJR spkelly@ntlworld.com

Competing interests: None declared.

1 Department of Health. Independent sector treatment centres. A report from Ken Anderson, Commercial Director London: Department of Health, 2006. www.dh.gov.uk/ assetRoot/04/12/91/08/04129108.pdf (accessed $10 \mathrm{Ma}$ 2006.

2 Royal College of Ophthalmologists. Response to the Health Select Committee. New inquiry-independent sector treatment centres. London: Royal College of Ophthalmologists, 2006. www.rcophth.ac.uk/docs/college/ RCOphthResponsetoSelectCommitteeInquiryonISTCs.pdf (accessed 10 Mar 2006.)

3 Kelly SP. Cataract care is mobile; is direction correct? $\mathrm{Br} \mathrm{J}$ Ophthalmol 2006;90:7-9.

4 Ferris JD. Independent sector treatment centres (ISTCs): early experience from an ophthalmology perspective. Ey 2005;19:1090-8.

5 Kelly SP. Recurring policy errors: blind spots over cataracts. Lancet 2005;366:1691.

\section{Untreated controls are wrong when proved treatment exists}

EDITOR-Mudur in his news article reports that Johnson \& Johnson had added that "the placebo controlled design was required to satisfy the requirements of regulatory authorities to allow their evaluation of the risk/benefit of the drug" in compliance with good clinical practice and the Declaration of Helsinki.

Article 29 of the Declaration of Helsinki statesthat thebenefits,risks,burdens,andeffectiveness of a new method should be tested against those of the best current prophylactic, diagnostic, and therapeutic methods. This does not exclude the use of placebo, or no treatment, in studies where no proved prophylactic, diagnostic, or therapeutic method exists.

The World Medical Association later added a footnote, saying that "a placebo controlled trial may be ethically acceptable, even if proved therapy is available ... Where for compelling and scientifically sound methodological reasons its use is necessary to determine the efficacy or safety of a prophylactic, diagnostic or therapeutic method."'

However, "the placebo controlled design was required to satisfy the requirements of regulatory authorities" is hardly a scientifically sound reason-it is a commercial one.

J Martin Bland professor of health statistics University of York, York YO10 5DD mb55@york.ac.uk

Competing interests: None declared.

1 Mudur G. Indian study sparks debate on the use of placebo in psychiatry trials. BMJ 2006;332:566. (11 March.)

2 World Medical Association. Declaration of Helsinki. Ethical principles for medical research involving human subjects. www.wma.net/e/policy/b3.htm (accessed 17 Mar 2006).

\section{Wear your name badge well}

Editor-Essex is right to point out the importance of introducing oneself in all clinical encounters. ${ }^{1}$ However, the position of the name badge may be acceptable at hip height, depending on where patients are seen, and in which specialty.

Despite my own short stature, most of my patients are around hip height, and so wearing my ID badge there is much easier for them. In hospital most ward rounds are conducted with the team standing, and so for patients lying or sitting in bed, badges at hip height are probably more easily read. In outpatient clinics most consultations occur while seated, and so a badge at breast height is more appropriate.

Wherever you wear your badge, the most important introduction and identification is with a clear voice.

Simon Fountain-Polley specialist registrar paediatrics Birmingham Heartlands Hospital, Birmingham B9 5SS

pollgysim@yahoo.com

Competing interests: None declared.

1 Essex C. Doctor who? BMJ 2006;332:583. (11 March.)

\section{bimj.com}

We select the letters for these pages from the rapid responses posted on bmj.com within five days of publication of the article to which they refer.

Letters are thus an early selection of rapid responses on a particular topic. Readers should consult the website for the full list of responses and any authors replies, which usually arrive after our selection. 